\title{
LES FACTEURS PRÉDICTIFS DE MALIGNITÉ DES NODULES THYROİDIENS À PROPOS DE 412 CAS
}

\author{
G. BESBES, N. BELTAIEF, M. OUKHAÏ, M. BEN MILED, S. TEMIMI, \\ S. TRABELSI, A. CHARFI, S. KHARRAT, S. SAHTOUT \\ SERVICE D'ORL ET DE CHIRURGIE CERVICO-FACIALE, HÔPITAL LA RABTA
}

\begin{abstract}
Introduction : Les nodules thyroïdiens constituent la pathologie thyroïdienne la plus fréquente. Ils posent surtout le problème de leur prise en charge. La recherche d'une étiologie cancéreuse reste la principale préoccupation.

Matériels et méthodes : Nous proposons une étude rétrospective, portant sur 412 patients porteurs de nodules thyroïdiens uniques ou multiples, colligés sur une période de 5 ans (1998-2002).

Nous avons réalisé une étude statistique comparant deux groupes de patients : le groupe I comprenant tous les patients porteurs de nodules malins pris en charge au cours de la période d'étude, ils sont au nombre de 103. Et le groupe II est constitué de 309 patients témoins pris au hasard porteurs de nodules bénins également pris en charge entre 1998 et 2002.
\end{abstract}

Résultats : Les facteurs prédictifs de malignité d'un nodule thyroïdien, jugés significatifs au terme de l'étude statistique univariée, étaient : l'âge inférieur à 30 ans, l'adénopathie révélatrice, la consistance dure du nodule, sa fixité, son caractère irrégulier et ses limites floues à la palpation, la présence d'une adénopathie à l'examen, l'association à une anémie. Et à l'échographie, la structure mixte, les limites irrégulières, la présence de microcalcifications et la visualisation d'une vascularisation intranodulaire avec ou sans vascularisation périnodulaire.

Seuls 4 facteurs étaient significatifs à l'étude multivariée : l'âge inférieur à 30 ans, l'anémie, l'échostructure mixte et l'hypervascularisation au doppler.

Conclusion : Bien que ces facteurs sont fortement prédictifs de malignité, aucun examen clinique ou paraclinique n'est suffisant à lui seul, le clinicien doit alors se baser sur un faisceau d'arguments pour adopter une conduite pratique parfois lourde de conséquences.

Mots clés : nodules thyroïdiens, malignité, dépistage, échographie

\footnotetext{
Introduction : Nodules are the most frequent feature in thyroid pathology. The screening of malignancy is the physician challenge.
}

Method : We report a retrospective study including 412 cases presenting a unique or multiple thyroid nodules diagnosed within 5 years (1998-2002) in the ENT department of La Rabta hospital of Tunis.

We achieved a statistic study comparing 2 groups of patients: the first one's size is 103 cases and included all patients presenting malignancy and treated in the same period. The second group included 309 patients presenting benign nodules chosen through a randomized way treated in the same period.

Results : The univariate study concluded as predictive an age inferior to 30 years, revealing nodes, hard consistency, fixed state, irregular shape, blurred limits and the existence of lymph nodes at palpation, and the association to anaemia. At ultrasonography, mixed aspect, irregular limits, microcalcifications and intranodular with or without peripheral vascularization.

Only 4 from these factors passed the multivariate study: age inferior to 30, anaemia, mixed aspect and intranodular vascularization.

Conclusion : Even if all these factors are predictive, no clinical or paraclinical feature is sufficient alone. The physician must collect all clinical and paraclinical predictive factors to adopt the treatment course to prevent malignancy without emphasizing the morbidity.

Key words : thyroid nodules, malignancy, screening, ultrasonography

\section{INTRODUCTION}

Les nodules thyroïdiens sont la pathologie thyroïdienne la plus fréquente. Leur prévalence est de 4 à $7 \%$ à l'examen clinique et atteint $30 \%$ à l'échographie.

La malignité de ces nodules est estimée à 10 à $20 \%$.

Le but de notre travail est de rassembler les critères épidémiologiques, cliniques et paracliniques prédictifs de malignité d'un nodule thyroïdien.

\section{MATÉRIEL ET MÉTHODES}

Notre étude est rétrospective type cas-témoins comportant 412 patients porteurs de nodules thyroïdiens opérés dans notre service entre 1998 et 2002.

Ont été inclus dans cette étude tous les patients présen- 
tant des nodules thyroïdiens uniques ou multiples diagnostiqués sur la clinique ou l'imagerie ; opérés au cours de la même période et ayant une histologie définitive. Les patients ont été subdivisés en deux groupes : Groupe I : il comprend tous les cas de nodules dont l'histologie définitive a conclu à la malignité. Il est composé de 103 patients.

Groupe II : il comprend 309 cas pris au hasard parmi les 1050 patients porteurs de nodules opérés au cours de la même période d'étude et dont l'histologie définitive est bénigne.

Les données épidémiologiques, cliniques, et paracliniques ont été incorporées dans une étude statistique univariée et multivariée permettant de chercher ceux dont la présence oriente significativement vers la malignité. L'intérêt de l'étude multivariée est de faire la part de risque (probabilité de malignité) entre deux ou plusieurs facteurs significativement liés entre eux.

\section{RESULTATS}

La fréquence de la malignité des nodules thyroïdiens dans notre série est de 9,8\% (103/1050). L'âge moyen varie selon les groupes. II est de 40,6 ans dans le premier groupe avec 2 pics sur la trentaine et la soixantaine et de 44,1 ans dans le 2ème groupe avec un pic sur la quarantaine. Une nette prédominance féminine a été relevée et ce dans les 2 groupes avec un sex-ratio de 7,6 dans le 1 er groupe contre 13 dans le 2ème.

Le nord-est et le nord-ouest sont les régions du pays les plus touchées que ce soit par la pathologie thyroïdienne cancéreuse que par la pathologie bénigne.

Parmi ces paramètres épidémiologiques, et selon les études statistiques, seul l'âge représente un facteur prédictif de malignité $(p=0,001)$. Les patients âgés de moins de 30 ans sont plus touchés par les nodules malins (28\%) versus $14 \%$ pour ceux âgés de plus de 30 ans.

Le sexe et l'origine géographique n'étaient pas des éléments d'orientation vers la malignité.

L'irradiation cervicale n'a été retrouvée chez aucun de nos cas. Onze $(2,7 \%)$ de nos patients ont subi une chirurgie thyroïdienne dans leurs antécédents et seul un d'entre eux a présenté un nodule malin. Nos observations n'ont pas relevé les antécédents obstétricaux de nos patientes. Ainsi, parmi les données personnelles étudiables de nos malades, aucune ne représente un facteur prédictif de malignité. Les antécédents familiaux de pathologie thyroïdienne bénigne $(12 \%)$ ou maligne $(17 \%)$ ne sont pas un facteur prédictif de malignité.

Le nodule thyroïdien était le motif de consultation le plus fréquent $(91 \%)$, découvert souvent par le patient ou son entourage et ce, autant pour les nodules malins que bénins. Les adénopathies «circonstances de découverte» étaient plus rencontrées dans les formes malignes (3,9\%) que bénignes. Les métastases à distance n'ont été révélatrices que dans un seul cas. Parmi ces motifs de consultation, seules les adénopathies révélatrices constituent un facteur prédictif de malignité $(p=0,02)$.

La durée d'évolution des nodules et la notion de poussée volumétrique rapide étaient similaires pour les 2 groupes. Les signes de compression, de dysthyroïdie, le flush syndrome et l'altération de l'état général étaient aussi non prédictifs de malignité.

Sur le plan clinique, les nodules touchaient autant les 2 lobes dans les 2 groupes et il n'y avait pas de différence significative entre le siège polaire supérieur ou inférieur. II était de même pour le caractère unique ou multiple. La taille moyenne des nodules malins était de $34 \mathrm{~mm}$ contre $33 \mathrm{~mm}$ pour les nodules bénins. La différence entre les deux est non significative ; la taille des nodules n'est donc pas un facteur prédictif de malignité. Cinq pourcent des nodules malins étaient mal limités à la palpation pour seulement $1 \%$ des nodules bénins. Les calculs statistiques ont conclu à une différence significative $(p=0,02)$. Le caractère mal limité d'un nodule à la palpation constitue alors un bon facteur prédictif de leur malignité. II en est de même pour le caractère fixé des nodules $(p=0,01)$, la consistance dure $(p=0,005)$ et le caractère irrégulier $(p<10-5)$. Par contre le caractère sensible à la palpation était plutôt rassurant et orientait vers la bénignité. La présence d'adénopathie à l'examen clinique était relevée dans $13 \%$ des nodules malins et seulement $3 \%$ des nodules bénins. La différence étant significative $(p=0,001)$, leur présence permet alors de prévoir le caractère malin des nodules. Le faible nombre de paralysie récurrentielle (1 seul cas) ne permet pas de conclure de sa valeur prédictive.

Bien que les malades porteurs de nodules malins avaient une tendance à l'hyperthyroïdie ( $8 \%$ versus $2,1 \%$ ), les tests statistiques étaient non significatifs. Aucun de nos patients n'avait une hypothyroïdie. Le dosage de la thyroglobuline, de la calcémie, de la calcitonine et de l'antigène carcino-embryonnaire n'était pas de pratique courante dans notre série. Par ailleurs on a relevé qu'une anémie est plus significativement vue chez les patients porteurs de nodules cancéreux que chez les autres $(p=0,014)$.

Les données relevées sur la radiographie du thorax, à savoir la déviation trachéale et les calcifications projetées sur l'aire thyroïdienne ne présentaient pas des éléments prédictifs de malignité.

En contre partie, il ressort de notre étude que l'échostructure mixte (38\% versus $32,8 \%)$, les contours irréguliers ( $7 \%$ versus $2 \%$ ), l'inexistence d'un halo périnodulaire (17\% versus $11 \%)$, l'existence de microcalcifications ( $11 \%$ 
versus $4,5 \%$ ) et la richesse de la vascularisation intranodulaire au doppler ( $3 \%$ versus $0,3 \%$ ) sont des facteurs échographiques prédictifs de malignité. Notre étude conclut aussi que le nombre, la taille, l'échogénicité des nodules et les végétations intranodulaires ne représentent pas des éléments de présomption de malignité.

Dans notre série, l'utilisation du scanner et de l'imagerie par résonance magnétique n'a pas été suffisante pour en tirer des conclusions statistiquement valables.

A la scintigraphie, le caractère froid était plus fréquent dans le groupe des nodules malins, mais il n'y avait pas de différence significative entre les 2 groupes $(p=0,12)$

Devant le nombre relativement faible de cytoponctions réalisées (4 cas), on n'a pas pu conclure de l'intérêt de cet examen dans le dépistage de la malignité.

Tous nos patients ont été opérés ( tableau I)

\begin{tabular}{|l|c|c|c|c|}
\hline & \multicolumn{2}{|c|}{ Nodules malins } & \multicolumn{2}{c|}{ Nodules bénins } \\
\hline & $\%$ & Nbre & $\%$ & Nbre \\
\cline { 2 - 5 } Thyroïdectomie totale & 61 & 63 & 31,4 & 97 \\
Loboisthmectomie & 34 & 35 & 64,4 & 119 \\
Thyroïdectomie subtotale & 4 & 4 & 30,6 & 11 \\
Isthmectomie & 1 & 1 & 0,6 & 2 \\
\hline Total & 100 & 103 & 100 & 229 \\
\hline
\end{tabular}

Tableau I : Gestes chirurgicaux thyroïdiens

L'évidement ganglionnaire a été pratiqué chez 97 patients dont 36 ont nécessité un geste en 2 temps. Chez les 6 patients restants, la malignité n'a été confirmée que sur I'histopathologie définitive. Le type histologique le plus fréquemment vu était le carcinome papillaire $(76,7 \%)$.

Parmi les 13 facteurs prédictifs de malignité retenus à l'étude univariée, seuls 4 ont passé le filtre de l'étude multivariée. Ces facteurs sont l'âge $<30$ ans, l'échostructure mixte, la vascularisation intra et périnodulaire à l'échographie et l'anémie.

\section{DISCUSSION}

La fréquence des nodules thyroïdiens est différemment estimée dans la littérature, les chiffres les plus avancés sont de 3 à $5 \%$, cette prévalence est encore plus élevée dans les séries autopsiques et échographiques (1). La fréquence du cancer varie entre 5 et $20 \%$ (2). Son incidence en Tunisie est estimée à 2,8 pour 100000 femmes et à 0,9 pour 100000 hommes selon le registre des cancers du nord tunisien (3).

Une nette prédominance féminine caractérise ce cancer avec un sex-ratio entre 3 et 4 (4). En revanche la littérature s'accorde sur le fait que le risque de malignité d'un nodule est plus élevé chez l'homme (5). II n'en est pas de même dans notre série.
Il est communément connu que le risque de cancer augmente pour les patients âgés de moins de 20 ans et ceux âgés de plus que 60 ans. Belfiore (5) trouve que le risque est maximal pour les âges extrêmes inférieurs à 30 ans et supérieurs à 60 ans. Dans notre série seul le jeune âge (< à 30 ans) constitue un facteur de risque.

On considère que le cancer de la thyroïde est plus fréquent dans les zones d'endémie goitreuse (6). Le registre des cancers du nord tunisien rapporte que le nord ouest est la région la plus touchée dans notre pays (3). Ceci vient à l'encontre des résultats de notre série qui rapporte que le nord-est est la zone où il y a le plus de risque de développer un cancer ; le nord ouest ne vient qu'en 2ème position. II est probable que cette discordance soit due à un biais de recrutement.

La littérature incrimine la TSH (7), les oestrogènes (8), les facteurs héréditaires (9), un régime pauvre ou trop riche en iode (6), les antécédents d'irradiation cervicale (10) et certaines pathologies thyroïdiennes bénignes tels que le nodule, le goitre, les thyroïdites, la maladie de Basedow et les dysthyroïdies (11)

Cliniquement, et comme dans notre série, la tuméfaction cervicale est le motif de consultation par excellence, estimée à $81,4 \%$ par Hugues et col. (12). Bien que parfois révélateurs, les signes de compression sont rarement rapportés dans la littérature (13). Par contre, la dysthyroïdie, les adénopathies cervicales et les métastases à distances rapportés comme circonstance de découverte sont de bons facteurs prédictifs de malignité selon les écrits (14). Selon Henry (15), la dysphagie et la dysphonie par paralysie récurrentielle témoignent plus d'un envahissement néoplasique que d'une simple compression et sont de ce fait forts évocateurs de malignité. II en est de même pour Hugues (12) qui rapporte que le risque de malignité est de $75 \%$ en cas d'association à une dysphonie et à une dysphagie. Cette association n'a pas été retrouvée dans notre étude. Le flush syndrome est aussi, selon certains auteurs, annonciateur de malignité. Néanmoins Bey-Boeglin (16) nous rappelle que l'absence de signes fonctionnels ne doit pas rassurer le clinicien d'autant plus que $73 \%$ des thyroïdes malignes sont asymptomatiques.

L'ancienneté des nodules ne doit pas, non plus, faire écarter leur potentiel malin, du fait de l'évolution à bas bruit des cancers (1). Bien que la plupart des auteurs s'accordent à insister sur l'aspect suspect des nodules qui augmentent rapidement en volume (17), leur stabilité ou leur évolution progressive ne doivent pas être rassurants comme cela a été confirmé dans notre série.

L'examen physique est capital dans le dépistage des nodules malins. L'aspect visible des nodules à l'inspection 
et la présence d'une circulation veineuse collatérale sont très suspects (18). Dans notre série comme dans celle de Tourniaire (19), le siège du nodule ne prédit pas de son caractère malin ou bénin. Certaines séries (5) on rapporté une prédilection du cancer pour le côté droit.

Notre étude et la plupart des séries de la littérature désapprouvent la notion de bénignité des goitres multinodulaires et rapportent que le risque néoplasique ne dépend pas du nombre de nodules (5). La taille clinique des nodules est de plus en plus dévalorisée par les auteurs en faveur des mensurations échographiques. De plus sa valeur pronostique est controversée. Une taille supérieure à $3 \mathrm{~cm}$ est jugée significative dans l'étude de Schlumberger (1) alors que Karagüsel (20) rapporte plutôt une taille inférieure à $2,5 \mathrm{~cm}$. La fixité, l'irrégularité et la consistance dure des nodules thyroïdiens représentent d'excellents facteurs pronostiques $(12,17)$. La sensibilité d'un nodule est une entité subjective et ne constitue pas un bon facteur pronostique (17). Par contre la découverte d'une paralysie récurrentielle homolatérale au nodule et d'adénopathie cervicale est très suspecte de malignité $(12,17)$.

Dans notre série comme dans la littérature, une dysthyroïdie n'est pas un facteur favorisant la malignité d'un nodule, cependant Tourraine (19) a rapporté une association de l'hyperthyroïdie avec les cancers thyroïdiens. L'anémie retrouvée comme bon élément prédictif de malignité dans notre série n'a pas été étudiée dans la littérature.

La radiographie cervicale a été délaissée du fait de son faible apport (12).

Sur la radiographie du thorax, seuls la découverte de métastases pulmonaires et/ou d'adénopathies médiastinales sont en faveur de la pathologie néoplasique (21).

Bien que l'échographie soit plus performante dans l'estimation de la taille d'un nodule, ce paramètre reste non significatif dans le dépistage des nodules cancéreux (2). Par contre l'aspect irrégulier est très évocateur de la malignité (22).

L'échostructure kystique, rare, représentant 5\% des nodules et reste un aspect échographique rassurant puisqu'il ne s'associe qu'à moins de $2 \%$ des carcinomes thyroïdiens (12). La forme hypoéchogène, la plus fréquente, est la plus pourvoyeuse de cancer thyroïdien $(23,24)$. Les aspects iso et hyperéchogènes bien que, s'associant à un moindre risque néoplasique, ne doivent pas être rassurants. Bien que l'échostructure mixte ne s'associe qu'à 10 à $20 \%$ des carcinomes thyroïdiens, tous les écrits s'accordent à dire que la plupart des cancers ont présenté cet aspect à l'exploration échographique. Naoum (24) publie même un chiffre de $92,9 \%$.
Les calcifications intranodulaires retrouvées à l'échographie correspondent à des lésions de calcosphérites dans les formes papillaires et médullaires, mais leur présence n'est pas pathognomonique de cancer (25). Leur intérêt dans le dépistage des cancers thyroïdiens est sujet à controverses du fait de leur « caractère opérateur dépendant ». Néanmoins les moyens utilisés actuellement permettent d'améliorer leur sensibilité. Leur spécificité élevée, estimée à 95,2\% pour Solbiati (26) permet de les classer parmi les éléments de présomption de malignité. Il en est de même pour le halo périphérique qui, s'il est incomplet, signe le cancer (26). Notre série s'accorde avec la littérature à conclure que la vascularisation intranodulaire associée ou non à une vascularisation périphérique au doppler signe la pathologie nodulaire néoplasique (27).

L'association d'adénopathies à l'échographie à des nodules thyroïdiens les rend suspects de malignité $(2,25)$. Elles sont volontiers sphériques, homogènes hypoéchogènes, sans hile et hypervascularisées au centre et en périphérie.

Le scanner cervical et l'imagerie par résonance magnétique sont plus indiqués dans l'exploration des goitres plongeants, les carcinomes anaplasiques et les lymphomes.

L'utilisation de la scintigraphie s'est nettement réduite ces dernières années. La littérature s'aligne sur le fait que la plupart des cancers thyroïdiens sont hypofixants (28), mais la majorité des nodules hypofixants sont bénins (19). Le caractère hyperfixant ne doit pas être rassurant comme il est communément admis ; Hugues (12) rapporte même un chiffre de malignité de $7,5 \%$.

La cytoponction thyroïdienne constitue de plus en plus un outil de dépistage des cancers thyroïdiens. Son avènement a permis de diminuer le nombre d'intervention de 25 à $50 \%$ et à augmenter l'incidence des cancers identifiés sur une pièce d'exérèse jusqu'à 30 ou même $40 \%$ (29). Elle peut se faire sur nodule palpable ou de façon échoguidée pour des nodules non palpables ou à composante mixte. Les études rapportent des résultats très disparates sur cet outil. Gharib (30) rapporte une sensibilité et une spécificité qui frôlent les 100\%. Ceci s'explique par le fait qu'elle est « opérateur et lecteur dépendante ». De même, depuis l'utilisation de l'immunocytochimie, sa sensibilité et sa spécificité ne cessent de s'améliorer.

\section{CONCLUSION}

II ressort de notre étude que l'âge inférieur à 30 ans, l'aspect clinique dur, fixé, irrégulier, mal limité et associé à des adénopathies cervicales, la présence d'une anémie, les contours irréguliers à l'échographie, la présence de 
microcalcifications, l'échostructure mixte, l'hypervascularisation intranodulaire associée ou non à un flux périnodulaire au doppler et la présence d'adénopathies à l'échographie représentent d'excellents éléments de dépistage des cancers thyroïdiens. Cependant une étude multivariée ne retient que l'âge inférieur à 30 ans, l'anémie, l'échostructure mixte et la présence d'un flux vasculaire comme facteurs prédictifs de malignité. Chaque temps du diagnostic est ainsi capital. Une meilleure appréciation des caractéristiques cliniques des nodules, les progrès de l'exploration paraclinique et surtout la généralisation de l'analyse cytologique et l'utilisation de l'immunocytologie permettront de mieux dépister les cancers afin de diminuer leur morbidité et d'éviter des interventions inutiles parfois lourdes de conséquences.

\section{REFERENCES}

1. Schlumberger R, Caillou B, Traglia JP et coll. Cancer de la thyroïde (à l'exclusion du cancer médullaire). Edition technique EMC. Gland Endoc Nutr 1990; 10008-A50-12:13

2. Castillo L, Haddad A, Meyer JM et coll. Facteurs prédictifs de malignité dans la pathologie nodulaire thyroïdienne. Ann Otolaryngol Chir Cervicofac $2000 ; 117$ :383-89

3. Registre des cancers du nord tunisien 1995-1998

4. Boudaya M, Germazi F, Drira MM. Cancers de la thyroïde. In : Maalej M. carcinologie pratique. Collection M/Sciences médicales 1999 : 199-208

5. Belfiore A, La Rosa GL, La Porta GA. Cancer risk in patient with cold thyroid nodes: relevance of iodine intake, sex, age and multinodularity. Am J Med 1992: 363-69

6. Belfiore A, Giacomo AB, La Rosa GL et coll. The frequency of cold thyroid nodules and thyroid malignancies in patients from an iodine-deficient area. Cancer 1987; 60: 3096-102

7. Winand RJ, Winand-Devigne J, Meurisse M. Effet de la thyrotropine (TSH) sur la prolifération cellulaire. La synthèse d'AMP-cyclique et l'incorporation d'iodure par les cellules d'adénocarcinomes thyroïdiens humains. Lyon Chir $1995 ; 91$ : 442-46

8. Inoue $\mathrm{H}$, Oshimo $\mathrm{K}$, Miki $\mathrm{H}$ et coll. Immunohistological study of estrogen receptors and the responsiveness to estrogen in papillary thyroid carcinoma. Cancer 1993; 72: 1364-68

9. Modigliani E. Le cancer médullaire de la thyroïde. Rev Prat $1996 ; 46$ : 230308

10. Schlumberger M, De Vathaire F. lode 131 : utilisation médicale. Effets cancérigènes et génétiques. Annales d'endocrinologie (Paris) 1996 ; 57 : 166-76 11. Belfiore A, Garofalo MR, Giuffrida D et coll. Increased aggressiveness of thyroid cancer in patients with graves's disease. J Clin Endocrinol Metab 1990; 70:830-35

12. Hugues FC, Baudet $\mathrm{M}$, Lacourreye $\mathrm{H}$ et coll. Le nodule thyroïdien : une étude rétrospective de 200 observations. Ann Otolaryngol 1989 ; $106: 77-81$ 13. Bennour M. Les nodules thyroïdiens à propos de 370 cas. Thèse de Médecine, Sousse 1992, $n^{\circ} 748$

14. Delisle MJ, Hannquin P, Liehn JC et coll. Les cancers thyroïdiens en Champagne-Ardenne : 1967-1984. exploitation épidémiologique d'un registre régional. Bull Cancer (Paris) $1986 ; 73: 165-70$

15. Henry JF. Commentaires sur l'évaluation clinique du nodule thyroïdien isolé. Annales d'Endocrinologie $1993 ; 54: 230-31$

16. Bey-Boeglin M, Dejax C, Thieblot $P$ et coll. Epidemiologic characteristics of
336 thyroid cancers observed in the Auvergne region. Annales d'Endocrinologie 1998; 59: 99-105

17. Cannoni $\mathrm{M}$, Dermand $\mathrm{F}$. Les nodules thyroïdiens du diagnostic à la chirurgie. Rapport de la société française d'otorhinolaryngologie et de pathologie cervicofaciale. Ed Arnette 1995 : 302 pages

18. Hermans J, Schmitz A, Merlo P et coll. Le Thallium 201 permet-il de différencier le nodule thyroïdien bénin du nodule malin? Annales d'Endocrinologie $1993 ; 54: 248-54$

19. Tourniaire J, Bernard MH, Mamelle $\mathrm{N}$ et coll. Nodules froids thyrö̈diens : étude prospective des critères de malignité chez 407 malades opérés. Nouv Presse Med 1981; $10: 309-12$

20. Kargüzel G, Tanyel FC, Büyükpamukçu $N$ et coll. Is there predictive characteristic for malignancy in thyroid enlargement during childhood? Eur J Pediatr Surg 1996; 6: 70-74

21. Kang KW, Kim SK, Kang HS et coll. Prevalance and risk of cancer of focal thyroid incidentaloma identified by $18 \mathrm{~F}$-Fluorodeoxyglycose positron emission tomography for metastasis evaluation and cancer screening in healthy subject. J Clin Endocrinol Metab 2003; 88: 4100-04

22. Leenhardt L, Dupasquier-Fediaevsky $\mathrm{L}$, Aurengo $\mathrm{H}$ et coll. Imagerie thyrö̈dienne. Encycl Med Chir (Elsevier, Paris), Endocrinologie-nutrition, 1999; 10002-F-10: 9p

23. Agence nationale pour le développement de l'évaluation médicale. Les recommandations pour la pratique clinique. La prise en charge du nodule thyroïdien. Ann Chir $1996 ; 50$ : 555-64

24. Naoum A. Evaluation de l'échographie dans le diagnostic des nodules thyroïdiens. Annales d'Endocrinologie 1993 ; 54 : 232-34

25. Bruneton JN, Marcy PY, Balu-Maestro C et coll. Les nodules thyroïdiens. Apport de l'imagerie. Feuillets Radiol $1993 ; 3$ : 1454-55

26. Solbiati L, Cioffi V, Ballarati E. Ultrasonography of the neck. Radiol Clin North Am 1992 ; 5: 941-51

27. Tramalloni J, Leger A, Correase JM et coll. Imagerie du nodule thyroïdien. J Radiol $1999 ; 80: 271-77$

28. Groussin P, Brizon J, Planiol Th et coll. Les nodules thyroïdiens: resultants de 320 observations. Sem Hop $1980 ; 56: 221-23$

29. Franc B, Allory Y, Hejblum G. la cytoponction des tumeurs de la thyroïde. Rev Prat 1996 ; 46 : 2315-20

30. Gharib H, Golner J. Fine-needle aspiration biopsy of the thyroid: an appraisal. Ann Intern Med 1993; 118: 282-89 